\title{
NOX4/NADPH oxidase expression is increased in pulmonary fibroblasts from patients with idiopathic pulmonary fibrosis and mediates TGF $\beta 1$-induced fibroblast differentiation into myofibroblasts
}

\author{
Nadia Amara, ${ }^{1}$ Delphine Goven, ${ }^{1}$ Fabienne Prost, ${ }^{1}$ Rachel Muloway, \\ Bruno Crestani, ${ }^{1,2}$ Jorge Boczkowski ${ }^{3,4,5}$
}

\begin{abstract}
- Supplementary materials and figures are published online only. To view these files please visit the journal online (http:// thorax.bmj.com).

'INSERM, Unité 700, Université Paris 7 Denis Diderot, site Bichat, Paris, France

${ }^{2}$ Assistance Publique-Hôpitaux de Paris, Service de

Pneumologie A, Hôpital Bichat, Paris, France

3 INSERM, Unité U955,

Université Paris Est, Faculté de Médecine, Créteil, F-94010,

France

${ }^{4}$ Assistance Publique-Hôpitaux de Paris, Service d'Explorations Fonctionnelles, Hôpital Henri

Mondor, Créteil, France

${ }^{5}$ Hôpital Intercommunal de Créteil, Service de Pneumologie et Pathologie Professionnelle, Créteil, France
\end{abstract}

Correspondence to Jorge Boczkowski, INSERM U955, Faculté de Médecine Paris 12, 94000 Créteil, France; jorge.boczkowski@inserm.fr

$\mathrm{BC}$ and JB shared senior authorship.

Received 7 January 2009 Accepted 25 May 2010

\section{ABSTRACT}

Background Persistence of myofibroblasts is believed to contribute to the development of fibrosis in idiopathic pulmonary fibrosis (IPF). Transforming growth factor $\beta 1$ (TGF $\beta 1$ ) irreversibly converts fibroblasts into pathological myofibroblasts, which express smooth muscle $\alpha$-actin $(\alpha-S M A)$ and produce extracellular matrix proteins, such as procollagen I ( $\alpha 1)$. Reactive oxygen species produced by NADPH oxidases (NOXs) have been shown to regulate cell differentiation. It was hypothesised that NOX could be expressed in parenchymal pulmonary fibroblasts and could mediate TGF $\beta 1$-stimulated conversion of fibroblasts into myofibroblasts.

Methods Fibroblasts were cultured from the lung of nine controls and eight patients with IPF. NOX4, $\alpha$-SMA and procollagen I ( $\alpha 1)$ mRNA and protein expression, reactive oxygen species production and Smad2/3 phosphorylation were quantified, in the absence and in the presence of incubation with TGF $\beta 1$. Migration of platelet-derived growth factor (PDGF)-induced fibroblasts was also assessed.

Results It was found that (1) NOX4 mRNA and protein expression was upregulated in pulmonary fibroblasts from patients with IPF and correlated with mRNA expression of $\alpha$-SMA and procollagen I ( $\alpha 1)$ mRNA; (2) TGF $\beta 1$ upregulated NOX4, $\alpha$-SMA and procollagen I ( $\alpha 1)$ expression in control and IPF fibroblasts; (3) the change in $\alpha$-SMA and procollagen I ( $\alpha 1)$ expression in response to TGF $\beta 1$ was inhibited by antioxidants and by a NOX4 small interfering RNA (siRNA); (4) NOX4 modulated $\alpha$-SMA and procollagen I ( $\alpha 1$ ) expression by controlling activation of Smad2/3; and (5) NOX4 modulated PDGF-induced fibroblast migration.

Conclusion NOX4 is critical for modulation of the pulmonary myofibroblast phenotype in IPF, probably by modulating the response to TGF $\beta 1$ and PDGF.

\section{INTRODUCTION}

Idiopathic pulmonary fibrosis (IPF) is the most common idiopathic interstitial pneumonia. IPF is a disease with poor prognosis and an aggressive nature, and poses major challenges to clinicians. ${ }^{1}{ }^{2}$ Currently, no effective treatments exist to stop ongoing fibrosis in IPF. Accumulation and persistence of myofibroblasts is believed to contribute to the development of fibrosis. ${ }^{3} \alpha$-Smooth muscle actin $(\boldsymbol{\alpha}$-SMA) expression, increased proliferative capacity, and increased generation and secretion of the extracellular matrix proteins such as collagen and fibronectin are key hallmarks of myofibroblast differentiation in fibrotic disorders. ${ }^{4}$ It has been established that myofibroblast differentiation occurs through combinatorial signals involving transforming growth factor $\beta 1$ (TGF $\beta 1$ ) and integrin signalling. ${ }^{5}$

Several studies suggest that oxidant-antioxidant imbalances in the lower respiratory tract play a significant role in the pathogenesis of IPF. Reactive oxygen species (ROS) can promote a profibrogenic environment in the lung through the modulation of the protease-antiprotease balance. ${ }^{6}$ Also, ROS activate and/or mediate the effects of growth-regulatory cytokines, including TGF $\beta 1 .^{7} 8$ However, the endogenous sources of oxidants in IPF are poorly known.

The NADPH oxidase (NOX) proteins generate ROS, namely superoxide, by electron transfer to oxygen. Superoxide can be further converted to hydrogen peroxide by cellular superoxide dismutases. NADPH oxidases consist of membraneassociated and cytosolic subunits (for review, see Lambeth ${ }^{9}$ ). The most thoroughly investigated $\mathrm{NADPH}$ oxidase is the phagocytic gp91 $1^{\text {phox }}$ (NOX2)-containing subunit that interacts with a second membrane-bound subunit, p22 ${ }^{\text {phox }}$, and several cytosolic and regulatory subunits including p47 $7^{\text {phox }}$, p67 $7^{\text {phox }}$ and Rac. Both NOX1 and NOX4 are homologues of NOX2, and are expressed in different non-phagocytic cells. ${ }^{9}$ It has been recently shown that NOX1 and NOX4 are expressed in pulmonary arterial adventitial fibroblasts and that NOX4 contributes to the increase in ROS generation under hypoxic conditions, stimulating proliferation and inhibiting apoptosis of these cells. ${ }^{10}$ Furthermore, NOX4 is involved in TGF $\beta 1$-induced human cardiac fibroblast differentiation into myofibroblasts. ${ }^{11}$ Therefore, we hypothetised that NOX1 and NOX4 could also be expressed in parenchymal pulmonary fibroblasts and could mediate the effects of TGF $\beta 1$.

The aim of this study was to investigate NOX1 and NOX4 expression in pulmonary fibroblasts from control subjects and patients with IPF, and to analyse their respective role in mediating TGF $\beta 1$-induced fibroblast differentiation into myofibroblasts, a key phenomenon in IPF pathogenesis. ${ }^{12}$ The myofibroblast phenotype was analysed by examining $\alpha$-SMA and procollagen I ( $\alpha 1)$ expression. 


\section{MATERIALS AND METHODS}

See online supplement for further details.

\section{Patients with lung fibrosis}

Fibroblasts were derived from lung tissue sampled from eight patients with IPF. Lung samples were obtained by open lung biopsy $(n=4)$ or at the time of lung transplantation $(n=4)$. IPF was diagnosed according to the American Thoracic Society/ European Respiratory Society (ATS/ERS) consensus criteria. ${ }^{13}$ Patients (six males; two females) had a median age of 50 years (range, 44-69 years). Six were ex-smokers, one never smoked and one was an active smoker. At the time of lung sampling, three patients were treated with low dose oral corticosteroids, associated with azathioprin in one patient.

\section{Control patients}

Fibroblasts were derived from lung samples from nine patients (six males, three females) undergoing lung surgery for removal of a primary lung tumour. Normal lung was obtained from a noninvolved segment, remote from the solitary lesion. Median age was 54 years (28-68 years). Two patients had never smoked, four were ex-smokers and three were active smokers.

\section{Culture of fibroblasts}

Human lung fibroblasts were cultured from lung explants until passage five as previously described. ${ }^{14}$ In some experiments, cells were incubated in the presence of TGF $\beta 1(10 \mathrm{ng} / \mathrm{ml}, \mathrm{R} \& \mathrm{D}$ Systems, Lille, France) or vehicle.

\section{Cellular toxicity}

Cellular toxicity and viability were assessed by trypan blue exclusion and lactate dehydrogenase $(\mathrm{LDH})$ release in the medium, as described previously. ${ }^{15}$

\section{Intracellular ROS production}

Intracellular ROS production was quantified by measuring $\mathrm{H}_{2}$-DCFH-DA (2,7-dichlorofluorescein diacetate) oxidation, as described previously. ${ }^{15} 16$ Results are expressed in fluorescent arbitrary units.

\section{Real-time reverse transcription-PCR (RT-PCR)}

NOX1, 2, 3, 4 and 5, $\alpha$-SMA and procollagen I ( $\alpha 1)$ mRNA expression was quantified by quantitative real-time RT-PCR by use of the PCR ABI 7700 apparatus (Applied Biosystems, Carlsbad, California, USA). Expression of mRNA was normalised to that of ubiquitin. In previous experiments, we verified that ubiquitin mRNA expression did not change under the different experimental conditions.

\section{Cell transfection with small interfering RNA (siRNA)}

To suppress endogenous NOX4 expression, experiments involved a specific siRNA (Xeragon-Qiagen, Valencia, California, USA,) against NOX4 designed on the target region from the NOX4 gene (5'-AAACCGGCAGGAGUUUACCCAG-3'). Scrambled siRNA (non-homologous to the human genome; 5'-AACCAGCAAGGUGUAUCGCCAC-3') was used as a control.

\section{Western blot analysis}

Western blot was performed as described previously ${ }^{17}$ with use of the following antibodies: polyclonal antihuman NOX4 (1/ 1000, Novus Biologicals, Littleton, Colorado, USA), monoclonal anti- $\boldsymbol{\alpha}$-actin (1/3000, Sigma-Aldrich, Saint Quentin Fallavier, France) and monoclonal antihuman anti-Smad2 and 3 (1/500, R\&D Systems, Lille, France). The expression of NOX4 was normalised in respect to the expression of $\beta$-actin. The expression of non-phosphorylated Smad2 (monoclonal antibody, 1/400, Cell Signalling, Saint Quentin Yvelines, France) was used to normalise the expression of phospho-Smad2/3.

\section{Cell migration assay}

Cell motility was examined as previously described ${ }^{18}$ using a modified Boyden chamber apparatus (Transwell Costar, Chorges, France) in response to $24 \mathrm{~h}$ stimulation with recombinant human platelet-derived growth factor-BB (rhPDGF-BB) $10 \mathrm{ng} / \mathrm{ml}$ ( $\mathrm{R} \& \mathrm{D}$ systems) or medium alone. The chemotactic index was determined as the ratio of cells moving in response to a chemoattractant relative to cells moving in response to medium control.

\section{Immunohistochemical analysis}

Immunohistochemistry was performed as previously described. ${ }^{17}$ Cryostat sections (4-6 $\mu \mathrm{m}$ thick) were fixed in acetone and reacted with the primary antibody rabbit polyclonal antiNOX4 (1/500 dilution, kindly provided by Professor Victor Thannickal, University of Alabama at Birmingham, Birmingham, Alabama, USA).

\section{Statistical analysis}

See online supplement for further details.

All the data are displayed as medians, IOR and minimum and maximum values. All of the analysis were performed by nonparametric tests. A p value $<0.05$ was considered as statistically significant.

\section{RESULTS}

\section{Expression of NOX mRNA and production of oxidants in} pulmonary fibroblasts

Lung fibroblasts from control patients expressed NOX1, 2, 4 and 5 mRNA to similar degrees, whereas expression of NOX3 was not detected in these cells (figure $1 \mathrm{~A}-\mathrm{D}$ ). When comparing IPF and control fibroblasts, NOX4 was the only NOX whose mRNA expression was increased in the former as compared with the latter condition $(p=0.043)$. The increased NOX4 expression in IPF fibroblasts was confirmed at the protein level by western blot ( $p=0.025$ control vs IPF, figure 1E). NOX4 mRNA and protein induction in IPF fibroblasts was accompanied by an increased intracellular ROS production in these cells as compared with control fibroblasts $(p=0.028$, figure $1 F)$. Immunohistochemistry performed on control and IPF lung samples demonstrated that lung fibroblasts expressed immunoreactive NOX4 in situ in the fibrotic lung (figure 2). Bronchial and alveolar epithelial cells and pulmonary endothelial cells also expressed immunoreactive NOX4.

\section{Expression of markers of myofibroblast diferentiation}

The content of $\alpha$-SMA mRNA was increased in IPF fibroblasts as compared with controls ( $p=0.045$; figure $\mathrm{E} 1$ in the online supplement) whereas procollagen I ( $\alpha 1)$ mRNA content was similar in both groups $(p=0.310$; figure E1 in the online supplement). Analysis of fibroblasts from patients with IPF showed a significant correlation between $\alpha$-SMA or procollagen I $(\alpha 1)$ mRNA and NOX4 mRNA expression (Spearman rho $=0.994$, $\mathrm{p}<0.0001$ in both cases; figure E1 in the online supplement), suggesting a role for this $\mathrm{NADPH}$ oxidase homologue in myofibroblast differentiation.

\section{Effect of TGF $\beta 1$}

Since NOX4 was overexpressed in IPF fibroblasts and correlated with markers of fibroblast differentiation into myofibroblast, 

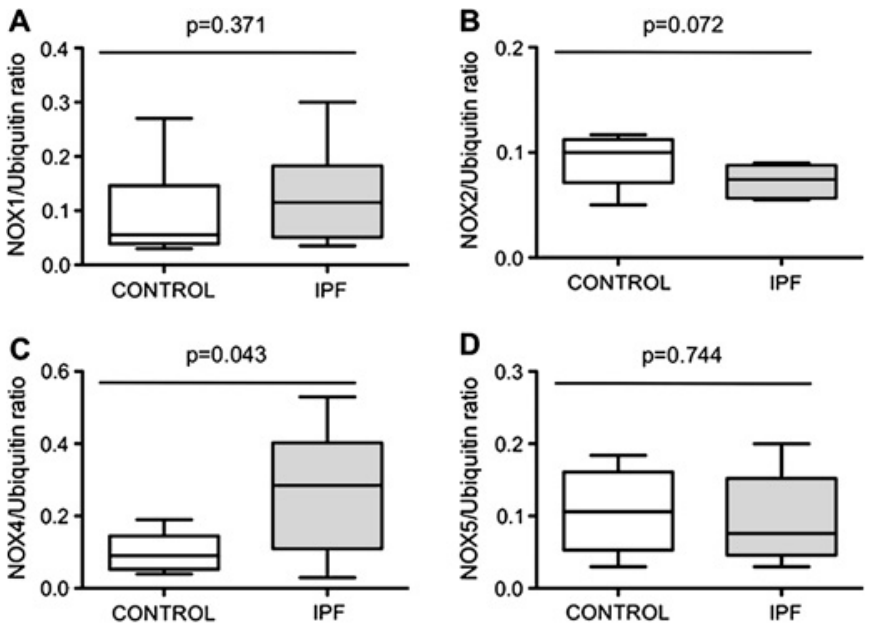

E
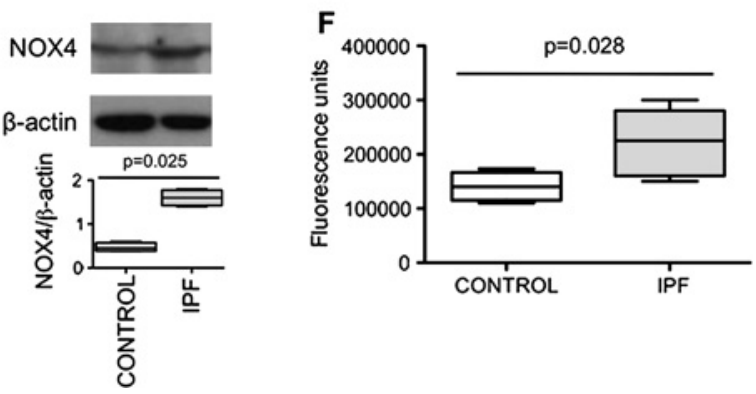

Figure 1 (A-D) NOX1, 2, 4 and 5 mRNA expression in lung fibroblasts from patients with idiopathic pulmonary fibrosis (IPF) compared with controls expressed as the ratio to ubiquitin mRNA levels. Data are presented as a box and whisker plot with median, IOR and minimum and maximum values. (E) Typical western blot and quantification of NOX4 protein expression (67 $\mathrm{kDa}$ ) in lung fibroblasts from patients with IPF and controls. NOX4 is expressed as the ratio to $\beta$-actin expression. Data are presented as a box and whisker plot with median, IQR and minimum and maximum values. (F) Reactive oxygen species production by IPF and control fibroblasts assessed by measurement of $\mathrm{H}_{2}$-DCFH-DA $(2,7$ dichlorofluorescein diacetate) oxidation. The values, expressed as arbitrary fluorescence units, are presented as a box and whisker plot with median, IQR and minimum and maximum values.

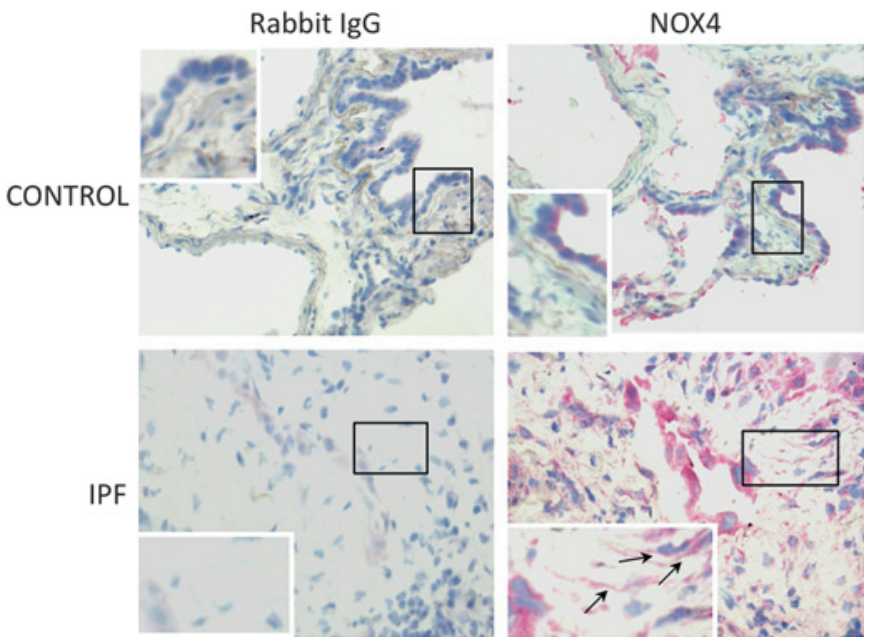

Figure 2 Detection of immunoreactive NOX4 in the lung. Immunohistochemistry shows that bronchial and alveolar epithelial cells express NOX4 in the normal lung. In idiopathic pulmonary fibrosis (IPF) lung samples, hyperplastic alveolar cells and fibroblasts (arrows) are strongly labelled. The control antibody always gave a negative signal. and since TGF $\beta 1$ is an essential inducer of myofibroblast differentiation, ${ }^{5}$ we analysed if TGF $\beta 1$ modulated NOX4 expression by lung fibroblasts. Incubation of control fibroblasts with TGF $\beta 1$ for $18 \mathrm{~h}$ induced a threefold increase of NOX4 mRNA content (figure $3 \mathrm{~A}, \mathrm{p}=0.014$ ). In IPF fibroblasts, TGF $\beta 1$ induced an eightfold increase of NOX4 mRNA content (figure 3B, $p=0.008)$. As expected, TGF $\beta 1$ increased $\alpha$-SMA and procollagen I $(\alpha 1)$ mRNA expression in control and IPF fibroblasts (figure $3 \mathrm{~A}, \mathrm{~B}, \mathrm{p}=0.009, \mathrm{p}=0.008, \mathrm{p}=0.034$ and $\mathrm{p}=0.029$ for $\alpha$-SMA in control and IPF fibroblasts and for procollagen I $(\alpha 1)$ in control and IPF fibroblasts, respectively). The increase in $\alpha$-SMA was also observed at the protein level (figure $3 \mathrm{C}$ ). Incubation of control fibroblasts with TGF $\beta 1$ induced a significant increase in ROS production, measured by oxidation of DCFH (figure 3D). This increase was more important in IPF than in control cells at 1,3 and $24 \mathrm{~h}$ post-TGF $\beta 1$ $(p=0.033, p=0.039$ and $p=0.028$ at each time, respectively, figure $3 \mathrm{D})$.

\section{Role of NOX4 in TGF $\beta 1$-induced myofibroblast differentiation}

We first examined if ROS, the product of NOX4 activity, were involved in TGF $\beta 1$-induced myofibroblast differentiation. First, we examined the effect of the antioxidant $N$-acetylcysteine (NAC). Pretreatment of control and IPF fibroblasts for $1 \mathrm{~h}$ with NAC $(1 \mathrm{mM})$ totally prevented the increase of $\alpha$-SMA and procollagen I ( $\alpha 1)$ mRNA expression induced by TGF $\beta 1$ (figure $3 \mathrm{~A}-\mathrm{C}$ ). This result strongly suggested that the oxidant-antioxidant imbalance is involved in the effect of TGF $\beta 1$.

In order to examine the involvement of NOX4 in TGF $\beta 1$ effects, we first incubated the fibroblasts for $1 \mathrm{~h}$ with diphenylene iodonium (DPI, $10 \mu \mathrm{M}$ ), an inhibitor of flavoprotein-dependent oxidases including NOX ${ }^{19}$ DPI prevented TGF $\beta 1$-induced $\alpha$-SMA and procollagen I $(\alpha 1)$ mRNA expression in both control and IPF fibroblasts (figure $3 \mathrm{~A}-\mathrm{C}$ ).

Secondly, we examined the effects of cell transfection with a NOX4 siRNA. NOX4 siRNA significantly reduced NOX4 mRNA levels at $24 \mathrm{~h}$ in both control and IPF fibroblasts, whereas no such effect was observed with lipofectamine or scrambled siRNA (figure 4A, B).

Transfection with NOX4 siRNA significantly inhibited the effect of TGF $\beta 1$ on myofibroblast differentiation in both control and IPF cells (figure 4A, B). Indeed, expression of $\alpha$-SMA and procollagen I ( $\alpha 1)$ was reduced by $67 \%$ and $68 \%$ and by $64 \%$ and $54 \%$ in control and IPF fibroblasts, respectively. These results demonstrate that NOX4 mediates the effect of TGF $\beta 1$ on myofibroblast differentiation.

It has to be noted that both NAC and DPI reversed NOX4 mRNA induction by TGF $\beta 1$, showing probably an autocrine role for NOX4-derived ROS in NOX4 induction by TGF $\beta 1$.

None of the different pharmacological treatments and transfection procedures altered cell viability (data not shown).

Role of NOX4 in TGF $\beta 1$-induced Smad2 and 3 phosphorylation It has been shown that Smads mediate TGF $\beta 1$ induction of $\alpha$-SMA in human lung fibroblasts. ${ }^{20} 21$ Since phosphorylation of Smad2/3 can be modulated by $\mathrm{ROS}^{11}$ we analysed whether NOX4 was involved in $\operatorname{Smad} 2 / 3$ phosphorylation induced by TGF $\beta 1$. The results of these experiments show that $1 \mathrm{~h}$ cell pretreatment with both NAC and DPI $(1 \mathrm{mM}$ and $10 \mu \mathrm{M}$, respectively) prevented $\mathrm{Smad} 2 / 3$ phosphorylation induced by $18 \mathrm{~h}$ incubation with TGF $\beta 1$ in control and IPF fibroblasts (figure 5A, B). Cell transfection with the NOX4 siRNA gave similar results (figure $5 \mathrm{~A}, \mathrm{~B}$ ). These results demonstrate that 

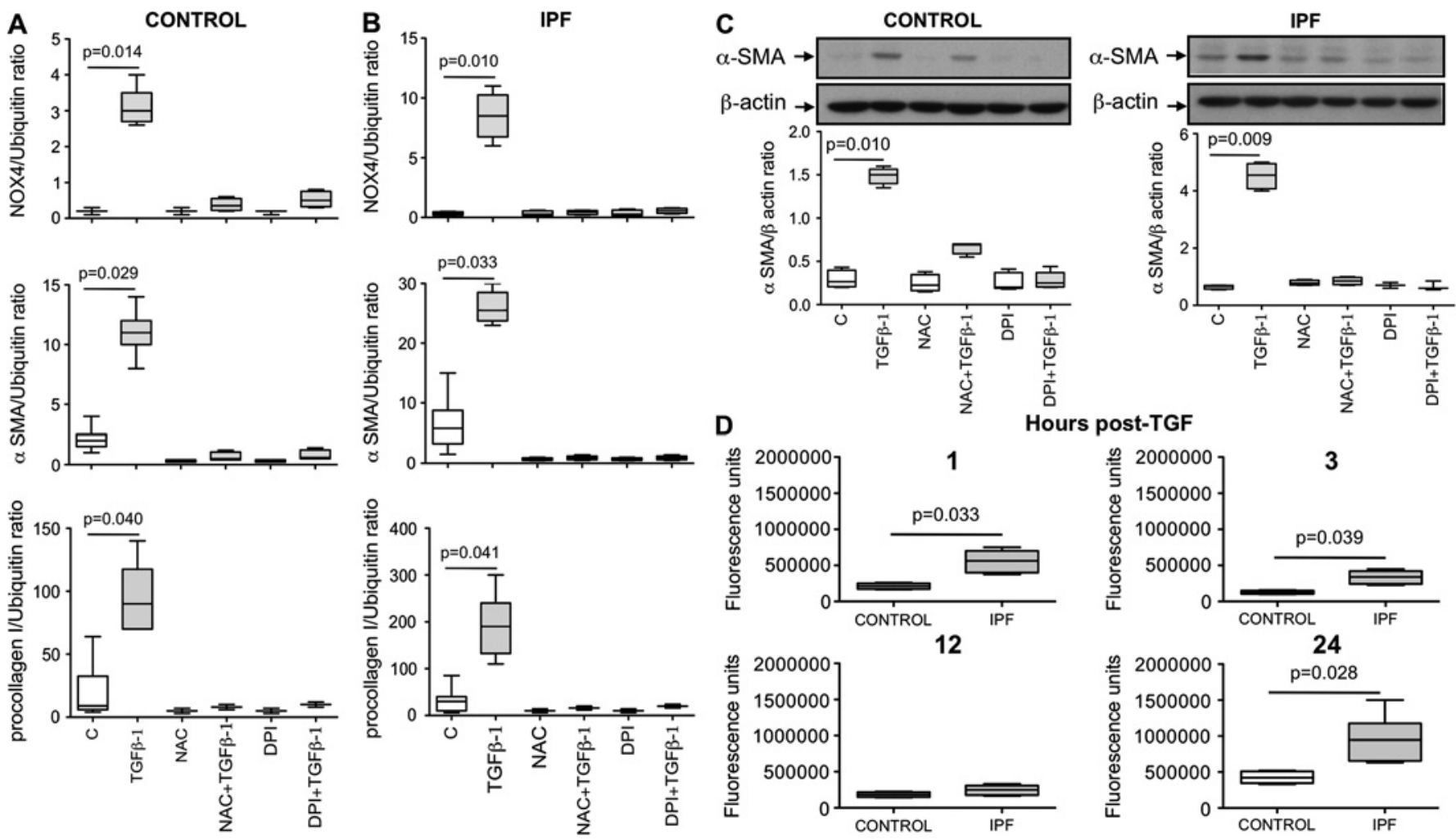

Figure 3 ( $\mathrm{A}$ and $\mathrm{B})$ Effect of transforming growth factor $\beta 1$ (TGF $\beta 1$ ) in the presence or in the absence of $N$-acetylcysteine (NAC, 1 mM) or diphenylene iodonium (DPI, $10 \mu \mathrm{m})$ on NOX4, $\alpha$-smooth muscle actin $(\alpha$-SMA) and procollagen I $(\alpha 1)$ mRNA expression in lung fibroblasts from controls and patients with idiopathic pulmonary fibrosis (IPF). Data are presented as a box and whisker plot with median, IQR and minimum and maximum values. (C) Typical western blot and quantification of $\alpha$-SMA protein expression in lung fibroblasts after TGF $\beta 1$ incubation in the presence or in the absence of NAC $(1 \mathrm{mM})$ or DPI $(10 \mu \mathrm{m})$. $\alpha$-SMA is expressed as the ratio to $\beta$-actin expression. Data are presented as a box and whisker plot with median, IQR and minimum and maximum values. (D) TGF $\beta 1$ stimulates reactive oxygen species (ROS) production in human lung fibroblasts. Control and IPF fibroblasts were stimulated with TGF $\beta 1$ for $1-24 \mathrm{~h}$ and ROS production was assessed by measurement of $\mathrm{H}_{2}$-DCFH-DA (2,7dichlorofluorescein diacetate) oxidation. The four figures show production by the two groups of cells at 1, 3, 12 and $24 \mathrm{~h}$ post-TGF $\beta 1$, respectively. Values are presented as a box and whisker plot with median, IQR and minimum and maximum values.

NOX4-produced ROS are involved in Smad2/3 phosphorylation by TGF $\beta 1$

\section{Role of NOX4 in PDGF-induced fibroblast migration}

Finally, we analysed if NOX4 was involved in fibroblast migration induced by PDGF-BB, a strong migration stimulus. First, we examined if PDGF modulated NOX4 expression by lung fibroblasts. Incubation of control fibroblasts and IPF fibroblasts with PDGF for $24 \mathrm{~h}(10 \mathrm{ng} / \mathrm{ml})$ induced a 1.5- and 2.5-fold increase of NOX4 mRNA content, respectively (figure $\mathrm{E} 2$ in the online supplement, $p=0.028$ and $p=0.002$, respectively).

Having demonstrated that NOX4 was upregulated by PDGF, we analysed its role in cell migration by incubating cells with NAC or DPI. Both NAC and DPI suppressed PDGF-induced fibroblasts migration in control and IPF fibroblasts, showing a role for NOX4 in this process (figure $\mathrm{E} 2$ in the online supplement and data not shown).

\section{DISCUSSION}

The main results of the present study are that (1) NOX4 mRNA expression was upregulated in pulmonary fibroblasts from patients with IPF and correlated with mRNA expression of $\alpha$-SMA and procollagen I ( $\alpha 1)$ mRNA; (2) TGF $\beta 1$ upregulates NOX4, $\alpha$-SMA and procollagen I ( $\alpha 1)$ mRNA and $\alpha$-SMA protein expression in control and IPF human pulmonary fibroblasts; (3) the change in $\alpha$-SMA and procollagen I ( $\alpha 1$ ) expression in response to TGF $\beta 1$ requires NOX4-derived ROS; (4) NOX4 modulates $\alpha$-SMA and procollagen I ( $\alpha 1)$ expression by controlling activation of Smad2/3; and (5) NOX4 modulates fibroblasts migration induced by PDGF-BB. These new findings support the notion that NOX4 is critical for modulation of pulmonary myofibroblast phenotype in IPF, probably by modulating the response to TGF $\beta 1$ and PDGF. Similar results concerning the response to TGF $\beta 1$ were very recently reported by Hecker and colleagues ${ }^{22}$ in an article published after the present study was submitted for publication.

\section{Expression of NOX4 in control fibroblasts}

Expression of NOX4 protein was detected in lungs from both control subjects and patients with idiopathic pulmonary artery hypertension; the expression was predominantly in the medial layer of pulmonary arteries and was higher in patients than in controls. ${ }^{10}$ Accordingly, pulmonary artery adventitial fibroblasts in culture also expressed both NOX1 and NOX $4 .{ }^{23}$ In agreement with these findings, in the present study we found that parenchymal pulmonary fibroblasts from controls and patients with IPF expressed both NOX1 and NOX4. This result is different from data reported in cardiac fibroblasts in which NOX1 expression was barely detectable, ${ }^{11}$ stressing the cell specificity of NOX expression. To the best of our knowledge this is the first demonstration that non-vascular pulmonary fibroblasts express NOX. It is noteworthy that our experiments were performed on 
A
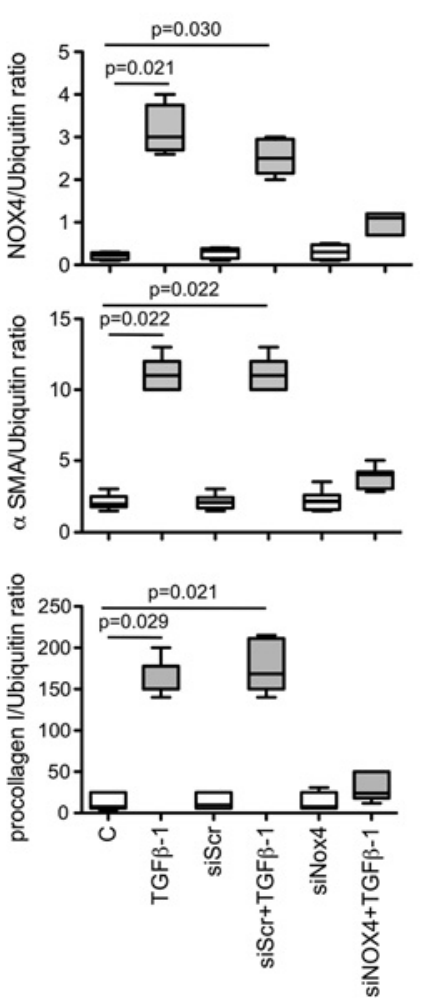

B
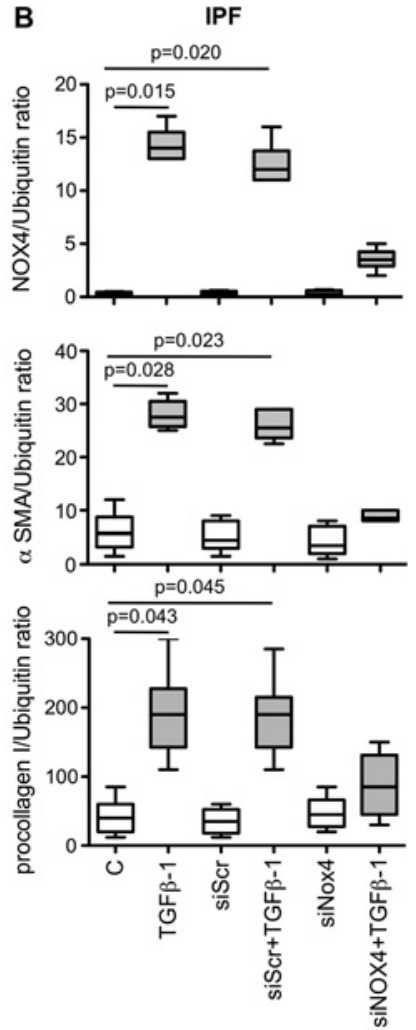

Figure $4 \quad(A$ and $B)$ Effect of cell transfection with a small interfering RNA (siRNA) anti-NOX4 on NOX4, $\alpha$-smooth muscle actin ( $\alpha$-SMA) and procollagen I ( $\alpha 1)$ mRNA expression induced by transforming growth factor $\beta 1$ (TGF $\beta 1$ ) in lung fibroblasts from controls and patients with idiopathic pulmonary fibrosis (IPF). SiScr, scrambled siRNA. NOX4, $\alpha$-SMA and procollagen I ( $\alpha 1)$ mRNA levels are expressed as the ratio to ubiquitin mRNA levels. Values are presented as a box and whisker plot with median, IQR and minimum and maximum values.

cells in the fifth passage, since progressive downregulation of NOX4 expression has been correlated with increasing passage number. ${ }^{24}$ However, our immunohistochemical results demonstrate that fibroblasts do express immunoreactive NOX4 in situ in the fibrotic lung.

\section{NOX4 upregulation in IPF fibroblasts}

The mechanism(s) involved in NOX4 upregulation in IPF fibroblasts are difficult to analyse since very few data concerning the regulation of NOX4 activity and expression are available. Recent data from recombinant NOX4 expression suggest that NOX4 enzymatic activity depends on the membrane-associated p22 $2^{\text {phox }}$ subunit, whereas cytosolic subunits, phosphorylation or interaction with Rac are apparently not required for its activation. ${ }^{25}{ }^{26}$ Concerning the regulation of gene expression, and the relevant transcription factors involved, very few data are available since promoter studies of NOX4 are lacking. Our data clearly show that TGF $\beta 1$ increases NOX4 expression both in control and in IPF fibroblasts, in accordance with previous data in cardiac fibroblasts and airway smooth muscle. ${ }^{1127} 28$ Interestingly, the increase was stronger in IPF fibroblasts than in controls. One can speculate that TGF $\beta 1$ can be responsible for NOX4 upregulation in IPF fibroblasts ex vivo via an autocrine loop, since expression of TGF $\beta 1$ has been shown to be increased in these cells. ${ }^{29}$ Furthermore, high levels of TGF $\beta 1$ produced by epithelial cells could contribute to upregulation in fibroblasts in situ in the lung of patients with IPF. ${ }^{30}$

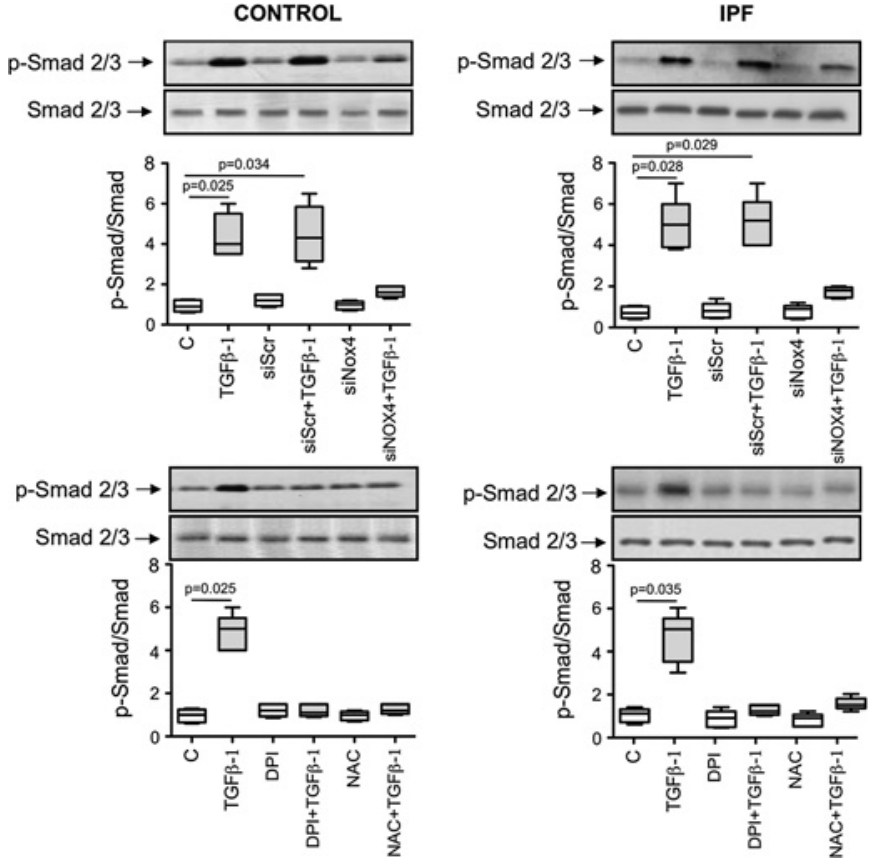

Figure 5 Typical western blot showing the expression of phosphorylated (p-Smad2/3) and non-phosphorylated Smad2/3 in human lung fibroblasts from controls and patients with idiopathic pulmonary fibrosis (IPF) after incubation with transforming growth factor $\beta 1$ (TGF $\beta 1$ ) in the presence or in the absence of a scrambled small interfering RNA (siScr), a siRNA anti-NOX4 (siNOX4), diphenylene iodonium (DPI, $10 \mu \mathrm{m}$ ) or $\mathrm{N}$-acetylcysteine (NAC, $1 \mathrm{mM}$ ). A Box and whisker plot with median, IQR and minimum and maximum values shows quantification of $p-S m a d 2 / 3$ expressed as the ratio of Smad2/3 expression.

NOX4 induction by TGF $\beta 1$ was inhibited by NAC and DPI, suggesting that NOX-produced ROS are implicated in NOX4 gene expression regulation. We obtained similar results in human alveolar bronchiolar and epithelial cell lines ${ }^{15}$ but the molecular mechanisms involved are unknown. Clearly, more studies are needed to understand NOX4 gene expression regulation in IPF fibroblasts.

\section{Role of NOX4 in myofibroblast differentiation in IPF}

Whatever the mechanism(s) involved in gene expression regulation, NOX4 was involved in myofibroblast differentiation in IPF. This conclusion is supported by (1) the correlation between basal NOX 4 and $\alpha$-SMA and procollagen I ( $\alpha 1)$ mRNA expression; and (2) the prevention of TGF $\beta 1$ differentiating effects by the NOX4 siRNA. This last phenomenon was also observed in cardiac fibroblasts. ${ }^{11}$ It has to be noted that we cannot ensure that NOX4-produced ROS were involved in myofibroblat differentiation in the present study since we did not measure fibroblast ROS production in NOX4 siRNA-treated cells. However, several pieces of experimental evidence strongly suggest that the effects we observed were mediated by ROS produced by NOX4. First, NOX4 was the only NOX homologue upregulated in IPF fibroblasts; secondly, ROS production was higher in these cells as compared with control cells; and, thirdly, similar results in terms of $\alpha$-SMA and procollagen I $(\alpha 1)$ expression were obtained by incubating these cell with the antioxidant NAC and by transfecting them with a NOX4 siRNA. Furthermore, a recent study with controlled recombinant expression of NOX4 demonstrated a strong correlation between NOX4 mRNA level and ROS generation. ${ }^{31}$ Thus, in this experimental setting, the activity of NOX4 was 
demonstrated to depend directly on its gene expression levels. Moreover, we and others have previously shown that siRNA against NOX4 significantly reduced the basal ROS level in rat smooth muscle cells and in pulmonary epithelial cell lines. ${ }^{15}$ Collectively, these data strongly support a role for NOX4-produced ROS in myofibroblast differentiation.

Interestingly, immunohistochemistry showed a strong expression of NOX4 by hyperplastic alveolar epithelium in the fibrotic lung (figure 2). In the kidney, NOX4 has been shown to be involved in the regulation of epithelial-mesenchymal transition. ${ }^{33}$ Although not specifically studied, this phenomenon could also occur in the lung and implicate NOX4 in the fibrogenic process through multiple pathways.

\section{Role of NOX4 in TGF $\beta 1$-induced myofibroblast differentiation and PDGF-induced migration}

TGF $\beta 1$ stimulates differentiation of fibroblasts into myofibroblasts using Smad2/3 signalling. ${ }^{21}$ After initial phosphorylation, Smad $2 / 3$ activation in response to TGF $\beta 1$ is prolonged (hours to days depending on the cell type). Interestingly, we showed that Smad2/3 phosphorylation was upregulated by TGF $\beta 1$ after $18 \mathrm{~h}$ of incubation. The antioxidant NAC, the NOX inhibitor DPI and the siRNA anti-NOX4 led to a marked inhibition of Smad2/3 phosphorylation, as demonstrated previously in human cardiac fibroblasts. ${ }^{11}$ These data imply that oxidants produced by NOX 4 mediate $\alpha$-SMA and procollagen I $(\alpha 1)$ upregulation in pulmonary fibroblasts in response to TGF $\beta 1$, probably by stimulating phosphorylation and activation of Smad2/3. In addition, NOX4 mediates PDGF-induced fibroblast migration.

In summary, we have shown NOX4 upregulation in pulmonary fibroblasts from patients with IPF, and identified a novel role for NOX4 as an essential mediator of $\mathrm{Smad} 2 / 3$ transcription factor activation in response to TGF $\beta 1$ in pulmonary fibroblasts. Because of the critical role of TGF $\beta 1$-induced myofibroblast activation in IPF, these data provide insights into novel mechanisms with potential therapeutic implications for IPF.

\begin{abstract}
Acknowledgements We acknowledge Françoise Zerah (Assistance PubliqueHôpitaux de Paris, Service d'Explorations Fonctionnelles, Hôpital Henri Mondor, Créteil, France) for statistical advice.
\end{abstract}

Funding NA was supported by Chancellerie des Universités de Paris (legs Poix), and JB by INSERM and Assistance Publique-Hôpitaux de Paris (Contrat d'Interface). This work was supported by the European Commission (FP 7, European IPF Network) and by the Agence Nationale de la Recherche (ANR Physio 2006, FIBROPNEUMO).

\section{Competing interests None.}

Patient consent Obtained.

Ethics approval This study was approved by the local ethics committee of Saint Germain en Laye Nospital and stored biopsies were reported to our institutional board (Délégation à la Recherche Clinique, Assistance Publique-Hôpitaux de Paris, Carré Historique de I'Hôpital Saint- Louis, Paris, France).

Provenance and peer review Not commissioned; externally peer reviewed.

\section{REFERENCES}

1. Dempsey 0J. Clinical review: idiopathic pulmonary fibrosis-past, present and future. Respir Med 2006:100:1871-85.

2. Maher TM, Wells AU, Laurent GJ. Idiopathic pulmonary fibrosis: Multiple causes and multiple mechanisms? Eur Respir J 2007;30:835-9.

3. Kuhn C, McDonald JA. The roles of the myofibroblast in idiopathic pulmonary fibrosis. Ultrastructural and immunohistochemical features of sites of active extracellular matrix synthesis. Am J Pathol 1991:138:1257-65.

4. Zhang K, Gharaee-Kermani M, McGarry B, et al. In situ hybridization analysis of rat lung alpha 1 (i) and alpha 2(i) collagen gene expression in pulmonary fibrosis induced by endotracheal bleomycin injection. Lab Invest 1994:70:192-202.
5. Serini G, Bochaton-Piallat ML, Ropraz P, et al. The fibronectin domain ed-a is crucial for myofibroblastic phenotype induction by transforming growth factor-beta1. J Cell Biol 1998;142:873-81.

6. Kinnula VL, Fattman CL, Tan RJ, et al. Oxidative stress in pulmonary fibrosis: a possible role for redox modulatory therapy. Am J Respir Crit Care Med 2005; 172:417-22

7. Yin L, Morita A, Tsuji T. Tobacco smoke extract induces age-related changes due to modulation of TGF-beta. Exp Dermatol 2003;12(Suppl 2):51-6

8. Amarnath $\mathbf{S}$, Dong L, Li J, et al. Endogenous TGF-beta activation by reactive oxygen species is key to Foxp3 induction in TCR-stimulated and HIV-1-infected human CD4+CD25- T cells. Retrovirology 2007:4:57

9. Lambeth JD. NOX enzymes and the biology of reactive oxygen. Nat Rev Immunol 2004; 4:181-9.

10. Mittal M, Roth M, Konig P, et al. Hypoxia-dependent regulation of nonphagocytic NADPH oxidase subunit NOV4 in the pulmonary vasculature. Circ Res 2007:101:258-67.

11. Cucoranu I, Clempus R, Dikalova A, et al. NAD(P)H oxidase 4 mediates transforming growth factor-beta1-induced differentiation of cardiac fibroblasts into myofibroblasts. Circ Res 2005;97:900-7.

12. Hinz B, Phan SH, Thannickal VJ, et al. The myofibroblast: one function, multiple origins. Am J Pathol 2007:170:1807-16.

13. American Thoracic Society. Idiopathic pulmonary fibrosis: diagnosis and treatment International consensus statement. American Thoracic Society (ATS), and the European Respiratory Society (ERS). Am J Respir Crit Care Med 2000:161:646-64.

14. Marchand-Adam S, Marchal J, Cohen M, et al. Defect of hepatocyte growth facto secretion by fibroblasts in idiopathic pulmonary fibrosis. Am J Respir Crit Care Med 2003:168:1156-61.

15. Amara N, Bachoual R, Desmard M, et al. Diesel exhaust particles induce matrix metalloprotease-1 in human lung epithelial cells via a NADP(H) oxidase/NOX4 redoxdependent mechanism. Am J Physiol Lung Cell Mol Physiol 2007;293:L170-81.

16. Thabut G, El-Benna J, Samb A, et al. Tumor necrosis factor-alpha increases airway smooth muscle oxidants production through a NADPH oxidase-like system to enhance myosin light chain phosphorylation and contractility. J Biol Chem 2002:277:22814-21.

17. Goven D, Boutten A, Lecon-Malas V, et al. Altered Nrf2/Keap1-Bach1 equilibrium in pulmonary emphysema. Thorax 2008;63:916-24.

18. Quesnel C, Nardelli L, Piednoir $\mathrm{P}$, et al. Alveolar fibroblasts in acute lung injury: biological behaviour and clinical relevance. Eur Respir J 2010;35:1312-21.

19. Hancock JT, White Jl, Jones OT, et al. The use of diphenylene iodonium and its analogues to investigate the role of the NADPH oxidase in the tumoricidal activity of macrophages in vitro. Free Radic Biol Med 1991;11:25-9.

20. Evans RA, Tian YC, Steadman R, et al. TGF-beta1-mediated fibroblast-myofibroblas terminal differentiation - the role of smad proteins. Exp Cell Res 2003:282:90-100.

21. Massague J. How cells read TGF-beta signals. Nat Rev Mol Cell Biol 2000:1:169-78.

22. Hecker $\mathrm{L}$, Vittal $\mathrm{R}$, Jones $\mathrm{T}$, et al. NADPH oxidase-4 mediates myofibroblast activation and fibrogenic responses to lung injury. Nat Med 2009;15:1077-81.

23. Li S, Tabar SS, Malec V et al NOX4 regulates ROS levels under normoxic and hypoxic conditions, triggers proliferation, and inhibits apoptosis in pulmonary artery adventitial fibroblasts. Antioxid Redox Signal 2008;10:1687-98.

24. Sturrock A, Cahill B, Norman K, et al. Transforming growth factor-beta1 induces NOX4 NAD(P)H oxidase and reactive oxygen species-dependent proliferation in human pulmonary artery smooth muscle cells. Am J Physiol Lung Cell Mol Physiol 2006;290:L661-73.

25. Ambasta RK, Kumar P, Griendling KK, et al. Direct interaction of the novel NOX proteins with p22phox is required for the formation of a functionally active NADPH oxidase. J Biol Chem 2004:279:45935-41.

26. Kawahara T, Ritsick D, Cheng G, et al. Point mutations in the proline-rich region of p22phox are dominant inhibitors of Nox1- and Nox2-dependent reactive oxygen generation. J Biol Chem 2005;280:31859-69.

27. Hu T, Ramachandrarao SP, Siva S, et al. Reactive oxygen species production via NADPH oxidase mediates TGF-beta-induced cytoskeletal alterations in endothelial cells. Am J Physiol Renal Physiol 2005;289:F816-25.

28. Sturrock A, Huecksteadt TP, Norman K, et al. NOX4 mediates TGF-beta1-induced retinoblastoma protein phosphorylation, proliferation, and hypertrophy in human airway smooth muscle cells. Am J Physiol Lung Cell Mol Physiol 2007;292. L1543-55.

29. Ramos C, Montano M, Garcia-Alvarez J, et al. Fibroblasts from idiopathic pulmonary fibrosis and normal lungs differ in growth rate, apoptosis, and tissue inhibitor of metalloproteinases expression. Am J Respir Cell Mol Biol 2001:24:591-8.

30. Khalil N, O'Connor RN, Flanders KC, et al. TGF-beta 1, but notTGF-beta 2 or TGF-beta 3 , is differentially present in epithelial cells of advanced pulmonary fibrosis: an immunohistochemical study. Am J Respir Cell Mol Biol 1996:14:131-8.

31. Serrander $L$, Cartier $L$, Bedard K, et al. Nox4 activity is determined by mRNA levels and reveals a unique pattern of ROS generation. Biochem J 2007;406:105-14.

32. Schroder K, Helmcke I, Palfi K, et al. Nox1 mediates basic fibroblast growth factor-induced migration of vascular smooth muscle cells. Arterioscler Thromb Vasc Biol 2007;27:1736-43.

33. Rhyu DY, Yang Y, Ha H, et al. Role of reactive oxygen species in TGF-beta1-induced mitogen-activated protein kinase activation and epithelial-mesenchymal transition in renal tubular epithelial cells. J Am Soc Nephrol 2005:16:667-75. 